\title{
Diversity of Abundance Patterns of Neutron-capture Elements in Very Metal-poor Stars*
}

\author{
Misa Aoki ${ }^{\dagger}$ \\ International Christian University \\ E-mail: g166016a@yamata.icu.ac.jp \\ Yuhri Ishimaru \\ International Christian University \\ E-mail: ishimaru@icu.ac.jp \\ Wako Aoki \\ National Astronomical Observatory of Japan \\ E-mail: aoki.wako@nao.ac.jp \\ Shinya Wanajo \\ RIKEN iTHES \\ E-mail: shinya.wanajo@riken.jp
}

\begin{abstract}
The astrophysical sites of $\mathrm{r}$ (apid) neutron-capture process are yet unclear. Chemical abundance patterns of very metal-poor stars have been studied to constrain the origin of the r-process, as these stars are believed to reflect the nucleosynthesis yields of a single or a few events. Recent observations have shown evidence that there are at least two components to r-process; "weak rprocess" mostly responsible for relatively light neutron-capture elements, and "main r-process" mostly responsible for heavier neutron-capture elements. A question is whether these two are well separated or there exists a variation in the r-process. This paper presents the results of abundance analysis of neutron-capture elements in five very metal-poor stars (HD 107752, HD 110184, HD 85773, HD 23798, BD +6 648) in the Milky Way halo observed with the Subaru Telescope High Dispersion Spectrograph. These stars show overabundance at lighter neutron-capture elements (e.g. Sr, Y), while heavier neutron-capture elements (e.g. Ba, Eu) are deficient, inferring element yielding of weak r-process. However, the overabundance of lighter ones is not as significant as that previously found in stars representing the weak r-process (e.g. HD 122563). Our study shows that there is diversity in the abundance patterns from light to heavy neutron-capture elements, which may depend on variation of electron fraction in supernova ejecta.
\end{abstract}

XIII Nuclei in the Cosmos,

7-11 July, 2014

Debrecen, Hungary

\footnotetext{
* Study based on data collected with Subaru Telescope, operated by the National Astronomical Observatory of Japan.

†'Speaker.
} 


\section{Introduction}

Nuclear astrophysics is a field with still many open questions; one big question is the sites of rapid neutron-capture process (r-process) in the Universe. Measurements of neutron-capture element abundances have been performed to very metal-poor stars in the Milky Way halo for investigations of nucleosynthesis process. Their elements are expected to be r-process origin and to reveal individual explosive nucleosynthesis processes.

Previous studies for numbers of metal-poor stars have revealed a distinctive feature in the abundance ratios between lighter neutron-capture elements and heavier ones. The ratio of these elements (e.g. [Sr/Ba]) show large scatter at low metallicity, which suggests variation in processing of lighter neutron-capture elements. One is referred to as "main r-process" (e.g. Truran et al. 2002), responsible for yielding of both heavy and light neutron-capture elements. It is considered to have a universal pattern, similar to that of solar $r$-process pattern. Another is the process referred to as "weak r-process", responsible for yielding of lighter neutron-capture elements. This process is thought to be the possible factor causing the large scatter of abundance ratios of lighter to heavier r-process elements at low metallicity. Precedent research projects which have observed several distinctive metal-poor stars support the existence of weak r-process. For example, lighter neutroncapture elements $(38 \leq \mathrm{Z} \leq 48)$ of HD 122563 are deviated (Honda et al. 2006; Wanajo \& Ishimaru 2006) compared to the abundance pattern of solar r-process. A question is, whether these two are well separated processes. An immediate question is whether the abundance pattern of weak r-process has diversity or universality as it has for the pattern of main r-process.

In order to obtain observational constraints on this problem, we analyze the abundance pattern of very metal-poor stars for further understanding of neutron-capture processes.

\section{Observation}

The high-resolution spectroscopy of our targets was carried out on February 8th and 9th, 2007, using the 8.2m Subaru Telescope High Dispersion Spectrograph (HDS). We selected five objects (HD 107752, HD 110184, HD 85773, HD 23798, BD +6 648), which are known to be very metalpoor $(-3 \leq[\mathrm{Fe} / \mathrm{H}] \leq-2)$ red giants that have enhanced light neutron-capture elements compared to heavy elements. The objects selected for our study have intermediate abundance ratios of $[\mathrm{Zr} / \mathrm{Ba}]$ between the two well-known objects, HD 122563 (Honda et al. 2006) and CS 22892-052 (Sneden et al. 2003). These stars are useful to study the variation of the yields of the process producing light neutron-capture elements at low metallicity. We obtained high resolution, high $\mathrm{S} / \mathrm{N}$ spectra for these objects with HDS. In addition, we used observational data from the Japanese Virtual Observatory (JVO) to determine the abundance of Ba II, since Ba has lines at longer wavelength

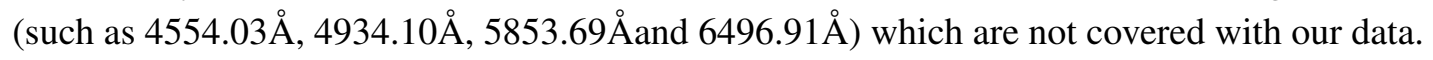

\section{Chemical Abundance Analysis}

We have performed abundance measurements on the basis of the model atmosphere and spectral line data, under the assumption of the local thermodynamic equilibrium (LTE). We adopt the model photosphere of Kurucz (1993) with a revised opacity distribution function (NEWODF) by 


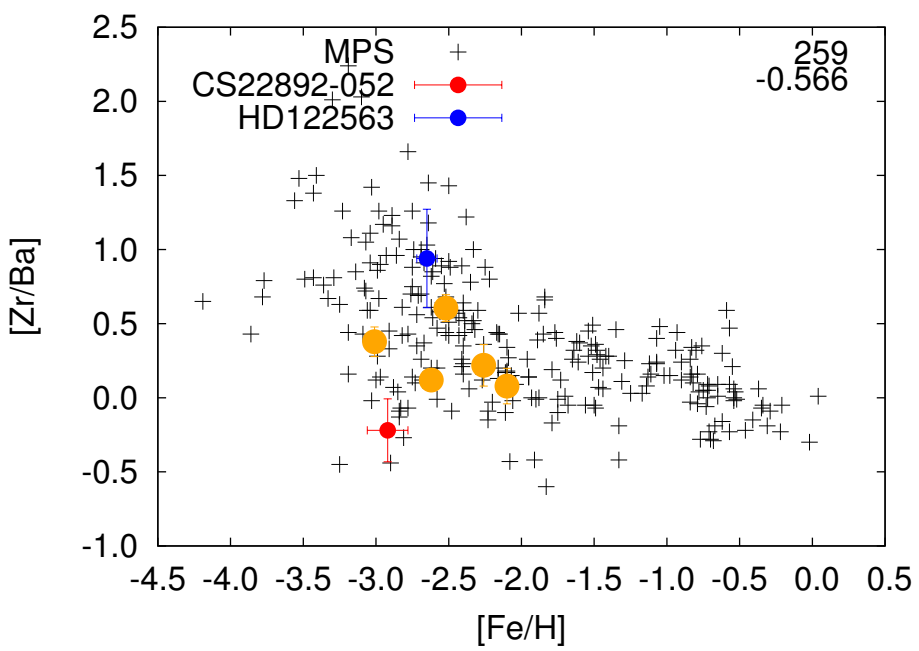

Figure 1: $[\mathrm{Zr} / \mathrm{Ba}]$ abundance ratios as a function of $[\mathrm{Fe} / \mathrm{H}]$. The black marks show data of halo stars. Red mark: CS22892-052; Blue mark: HD 122563; Orange points: Object stars measured in the present work

Castelli \& Kurucz (2003). The parameters of model atmospheres $\left(T_{\text {eff }}, \log g\right)$ are adopted from previous studies (e.g. Ishigaki et al. 2009). Line data is taken from Honda et al. (2006)

For elements with several absorption lines available (Y II, Zr II, Ba II, La II, Ce II, and Nd II) in our spectra, abundances are measured for individual lines and their average is taken. On the other hand, fitting observed spectrum with a calculated one (spectrum synthesis method) is applied to determine abundances of elements with few measurable lines (Sr II, Mo I, Ru I, Pd I and Eu II). Spectrum synthesis method is taken for lines affected by contamination or for those elements with one or two measurable lines. A total of 11 elements are measured for each very metal-poor star. Abundances of $\mathrm{Sr}, \mathrm{Y}$ and $\mathrm{Zr}$ have been determined by previous researches for HD 110184, but Mo, $\mathrm{Ru}$ and Pd lines are measured for the first time. For HD 107752, HD 85773 and HD 23798, Sr is also measured for the first time.

The size of random errors in the measurements is estimated from the standard deviations of the abundances derived from individual lines. For the abundances of elements that have only one or two available lines, we employ value of $0.1 \mathrm{dex}$, which is an estimated mean value of the random errors of those elements with three or more lines.

\section{Results}

\subsection{Abundance Pattern}

The chemical abundances of target stars determined by the present work are compared with the solar r-process pattern taken from Simmerer et al. (2004). Figure 2 shows examples of comparisons between stellar abundance pattern and solar r-process pattern. The left chart of Fig. 2 shows comparison of the abundance of HD 107752 with the solar r-process value. At the heavier neutroncapture elements, the pattern resembles that of the solar r-process. On the other hand, abundance of light neutron-capture elements show overabundance compared to the solar r-process pattern. This overabundance is not as evident as HD 122563, but suggests that the object star has weak r-process 

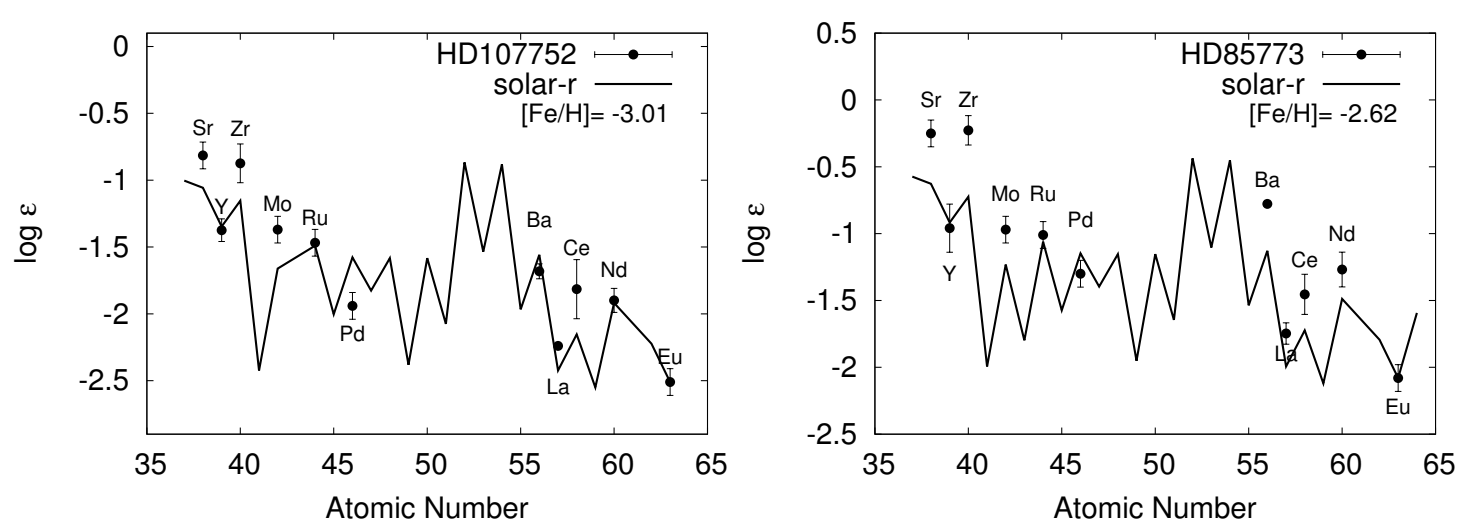

Figure 2: Abundance patterns of HD 107752 (left) and HD 85773 (right) from strontium to europium compared with the solar r-process pattern (solid lines).

contribution. HD 110184 has an abundance pattern similar to that of HD 107752, likewise having evidence of slight weak r-process contribution.

HD 85773 (right figure), BD +6 648, and HD 23798 also exhibit overabundances of light neutron-capture elements compared to the solar r-process pattern. We note that heavier neutroncapture elements, especially barium, are also overabundant for these three stars, suggesting contamination of s-process to the heavier neutron-capture elements. We measured the abundance of lead $(\mathrm{Pb})$, which is an element known to be enriched by s-process nucleosynthesis, in particular at low metallicity, using the Pb I $3683.46 \AA$ line. The result shows that the abundance of lead is low, which implies that s-process contamination is not severe. The lead abundances of the three stars, BD +6648, HD 85773 and HD 23798, are $\log \varepsilon \approx-0.25$, which would be explained by a small contribution of the s-process.

Since the s-process at low metallicity produces few light neutron-capture elements, overabundance of lighter neutron-capture elements would be explained by contamination of weak r-process rather than s-process.

\subsection{Comparison of Lighter Neutron-capture Elements}

Figure 3 shows abundance patterns of lighter neutron-capture elements from strontium to palladium of five observed stars. The pattern of HD 122563 is also shown as an example of weak r-process enhanced star. The abundance pattern of HD 107752 and HD 110184 is similar to that of HD 122563. We suggest that this pattern is a representative pattern of weak r-process. The other three objects show drastic drops towards palladium. The drop cannot be replicated by combination of the abundance pattern expected for the main r-process and the representative pattern of the weak r-process because neither show such rapid drops in their abundance pattern. Therefore, we infer that the abundance pattern of lighter neutron-capture elements produced by weak r-process have diversity.

\section{Summary and Discussion}

We have investigated the abundance pattern of neutron-capture elements in five metal-poor 


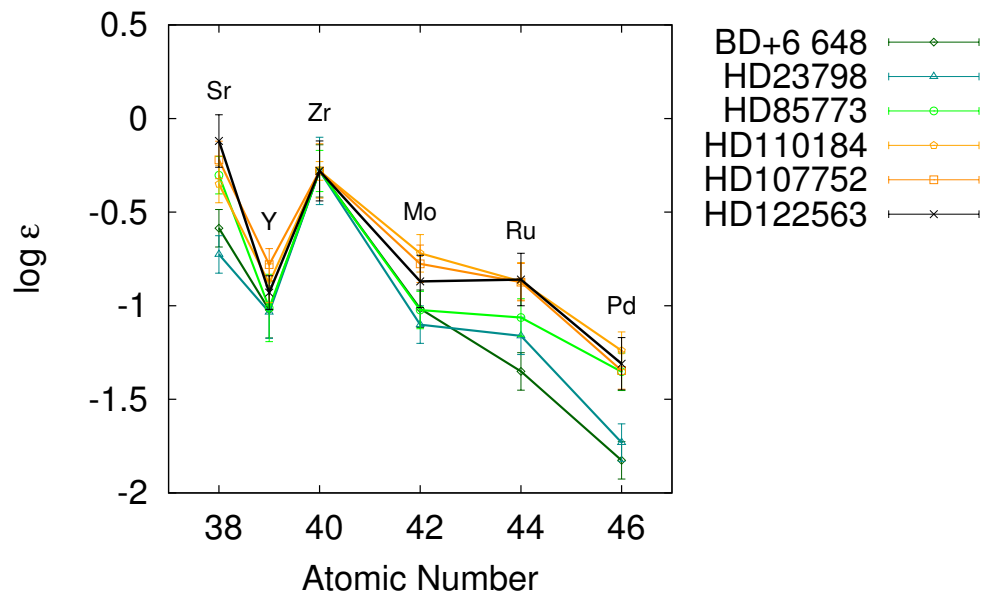

Figure 3: Abundance patterns of lighter neutron-capture elements from strontium to palladium of five observed stars and HD 122563 as a sample of weak r-process enhanced star. Object patterns are shifted to zirconium of HD 122563 (black line).

stars. Some of the elements, such as ruthenium and molybdenum, are measured for the first time.

Our objective is to know whether the abundance pattern of weak r-process has diversity or universality. According to our comparison of abundance patterns, weak r-process has diversity in the abundance patterns even within lighter neutron-capture elements. A possible explanation to this variation of r-process is variation of $Y_{e}$ (electron fraction) in electron capture supernova ejecta. Wanajo et al. (2011) have performed simulation of elemental abundances for various $Y_{e}$. Their simulation showed different abundance patterns depending on the value of $Y_{e}$; for example, when the value of $Y_{e}$ is high (neutron fraction is low), the abundance pattern shows a significant drop from lighter neutron-capture elements towards heavier neutron-capture elements.

Further observation and analysis are required to determine the size of scatter in the abundance patterns, which would be useful to constrain the astrophysical sites of r-process.

\section{References}

[1] Castelli, F. \& Kurucz, R. L. 2003, IAUS, 210P, A20

[2] Honda, S., Aoki, W., Ishimaru, Y., Wanajo, S. \& Ryan, S. G. 2006, AJ, 643, 1180-1189

[3] Ishigaki, M., Chiba, M. \& Aoki, W. 2009, arXiv:0912.0329

[4] Sneden, C., Cowan, J. J., Lawler, J. E., Ivans, I. I., Burles, S., Beers, T. C., Primas, F., Hill, V., Truran, J. W., Fuller, G. M., Pfeiffer, B. \& Kratz, K. 2003, ApJ, 591, 936

[5] Simmerer, J., Sneden, C., Cowan, J. J., Collier, J., Woolf, V.M. \& Lawler, J. E. 2004, ApJ, 617:1091-1114

[6] Truran, J. W., Cowan, J. J., Pilachowski, C. A. \& Sneden, C. 2002, PASP, 114, 1293

[7] Wanajo, S., \& Ishimaru, Y. 2006, Nucl. Phys. A, 777, 676

[8] Wanajo, S., Janka, H.-Th. \& Müller, B. 2011, ApJ, 726, L15 